\title{
Lessons from the Real World: Financial Incentives to Improve Glycemic Control in Patients with Type 2 Diabetes
}

\author{
June F. O'Leary¹, Janelle Howe², Jeremy Rich ${ }^{3}$, Glenn Melnick ${ }^{1}$ \\ ${ }^{1}$ Sol Price School of Public Policy, University of Southern California, Los Angeles, CA, USA \\ ${ }^{2}$ DaVita HealthCare Partners Medical Group, El Segundo, CA, USA \\ ${ }^{3}$ HealthCare Partners Institute for Applied Research and Education, El Segundo, CA, USA \\ Email: gmelnick@usc.edu
}

How to cite this paper: O'Leary, J.F., Howe, J., Rich, J. and Melnick, G. (2018) Lessons from the Real World: Financial Incentives to Improve Glycemic Control in Patients with Type 2 Diabetes. Health, 10, 171-180. https://doi.org/10.4236/health.2018.102014

Received: January 12, 2018

Accepted: February 8, 2018

Published: February 11, 2018

Copyright $\odot 2018$ by authors and Scientific Research Publishing Inc. This work is licensed under the Creative Commons Attribution International License (CC BY 4.0).

http://creativecommons.org/licenses/by/4.0/

\section{c. (i) Open Access}

\begin{abstract}
Objectives: While the value of glycemic control to minimize adverse health outcomes among patients with diabetes is clear, achieving hemoglobin A1c (A1c) goals remain a challenge. We evaluated the use of financial incentives to increase enrollment and improve glycemic control among patients invited to participate in a monthly diabetes group appointment (DGA) as part of their enrollment in DaVita HealthCare Partners, a large southern California managed care organization. Methods: Adult diabetes patients ( $\geq 18$ years) with a currently uncontrolled hemoglobin A1c level $(>8.0 \%$ if $<65$ years and $>9.0 \%$ if $\geq 65$ years) were randomized to 1) no DGA, 2) DGA with no financial incentives (non-incentive DGA) or 3) DGA with financial incentives (incentive DGA). Results: Nine sites among four regions of the greater Los Angeles area participated. Each site offered one non-incentive DGA and one incentive DGA. Over 1500 patients were identified for recruitment and at the peak of enrollment, 299 patients were enrolled in 18 DGAs. On average, hemoglobin Alc values dropped more for patients participating in the incentive DGA (9.9\% to $8.7 \%,-1.2 \%)$ versus non-incentive DGA $(9.7 \%$ to $9.0 \%,-0.7 \%)$ versus no DGA group $(9.1 \%$ to $8.7 \%,-0.4 \%)$. Several unexpected implementation challenges arose which complicated evaluation but provide important learning lessons. Conclusions: Management of chronic diseases like diabetes is challenging for patients and the primary care system alike. Continuing to implement and evaluate programs under "real-world" conditions can provide further insight into how best to support patients with diabetes and their primary care teams in order to achieve glycemic control and avoid preventable complications.
\end{abstract}




\section{Keywords}

Diabetes, Glycemic Control, Financial Incentives, Behavioral Economics, Group Appointments

\section{Introduction and Background}

It is estimated that 23 million people are living with a diabetes diagnosis in the U.S. with similar rates in California (2.5 million adults with diabetes, 9\%) [1]. Another 13 million Californians are estimated to have undiagnosed diabetes or prediabetes, a high risk state for developing diabetes [2]. Diabetes is the $7^{\text {th }}$ leading cause of death in the U.S. with total direct and indirect costs of $\$ 245$ billion in 2012 [1]. The burden of diabetes is clear.

Studies have also shown that patients with diabetes who control their blood glucose (as measured by their hemoglobin A1c level) can prevent or delay microvascular complications like eye and kidney disease, but that doing so is challenging [3]. Type 2 Diabetes is a chronic disease that requires patient self-management in the form of diet and exercise, and sometimes medication in order to achieve glycemic control. The importance of diabetes self-management education and support (DSMES) is underscored by the National Standards which seek to guide the provision of timely, evidence-based DSMES programs [4]. Primary care teams at DaVita HealthCare Partners (DHCP) implemented diabetes group appointments (DGA) to educate, support and monitor patients in the self-management of their diabetes in order to prevent or delay complications.

Group appointments show promise to efficiently deliver high quality primary care, education and support to chronic disease patients, including those with diabetes [5] [6] [7]. DHCP staff indicated some success among patients attending DGAs, but felt the potential of the DGAs was not being reached. Primary care teams aimed to increase DGA participation and help patients reach their A1c goal. DHCP sought to implement more DGAs, including DGAs with financial incentives, with the purpose of increasing participation and improving patient A1c levels. Researchers at the University of Southern California (USC) were consulted to provide feedback on how DHCP might design, implement and evaluate the DGAs with financial incentives in order to assess their effectiveness.

The literature regarding the application of behavioral economic principles to change health behaviors is relatively large at this point (for example see https://ldi.upenn.edu/research-interest/behavioral-economics-behavior-change and [8]), but empirical studies of the impact of financial incentives on diabetesrelated outcomes is much smaller. A recent scoping review of behavioral economic interventions for the prevention and treatment of Type 2 diabetes uncovered six studies that tested the impact of financial incentives on either selfmanagement recommendations or outcomes [9]. Only two studies specifically addressed using financial incentives to reach Alc goals and the results were 
modest at best [10] [11].

The objective of this study was to provide guidance to DHCP on how primary care teams could feasibly test the impact of financial incentives on DGA attendance and Alc outcomes. Group appointments require significant resources and a rethinking if not a redesign of the traditional primary care system [12] [13]. Study findings offer insights for the primary care community both with respect to the potential ability of economic incentives to engage patients in management of their chronic condition as well as valuable lessons on how to structure, implement and evaluate interventions in a primary care setting.

\section{Methods}

\subsection{Intervention: Diabetes Group Appointments (DGAs) with and without Financial Incentives}

Diabetes group appointments were developed for patients who have not achieved glycemic control. Group appointments can effectively target primary care resources when caring for patients with chronic disease, including diabetes [5] [6] [7]. The main goal of DHCP's DGA is to help each patient achieve glycemic by increasing their understanding of their condition and facilitating engagement and behavior change through an active team approach, including peers.

The main characteristics of the DGAs include:

- 2.5 hour free group appointment

- About 10 patients per group

- Monthly, same day and time each month

- In English or Spanish

- Providers involved:

o Health educator(s)

o Registered dietician or certified diabetes educator

o Registered nurse or certified diabetes educator

o Physician (primary care or endocrinologist)

- Medical assistant(s)

At the first DGA session clinical values are checked including A1c, fasting blood glucose, LDL, weight and blood pressure and a foot check and PHQ-9 to screen for depression are done [14]. Follow-up foot checks are conducted every six months, A1c and LDL levels are checked every three months, and the remaining measures at every DGA or monthly. A healthy breakfast is provided as part of a meal preparation demonstration.

Next, the structured educational portion of the DGA begins and at the first DGA an action plan is developed that addresses barriers to health such as lack of physical activity, smoking and stress. Each DGA covers a predetermined topic including "Monitoring Your Blood Glucose" and "Diabetes Nutrition with a Registered Dietician." When a patient reaches their A1c goal they graduate from the DGA. For patients age 65 years and younger this is generally considered an 
A1c $<7.0 \%$ and for patients age 65 years and older an A1c $<8.0 \%$ [3]. At their last DGA, graduates are recognized and given a certificate and diabetes-appropriate gift basket (e.g., book about managing diabetes, diabetes cookbook, measuring cups and spoons, brown rice, healthy seasonings). Patients are then invited to join a diabetes support group.

In the case of financial incentives, these were provided in three ways:

1) $\$ 40$ incentive to attend first DGA meeting

2) $\$ 40$ incentive for each $0.5 \%$ reduction in A1c level until goal

3) Chance to win $\$ 40$ at each DGA meeting

While these financial incentives derive from the literature cited earlier, these incentives were also developed with input from DHCP primary care teams. Certain themes recurred:

- The importance of an incentive to get people to the first meeting, so that patients can see the benefit and form bonds with both the primary care team and peers.

- Positive incentives and fun atmosphere.

o Loss aversion may be a powerful incentive, but concerns about alienating patients or the development of negative attitudes were expressed [8].

o Staff viewed incentives tied to A1c reductions as valuable, they also felt DGA attendance in and of itself was useful. A wheel was spun at the end of each meeting. Each equal "slice" on the wheel included an attendee's name and this is how a meeting "winner" was determined.

- Keep the incentive schemes simple, to ease implementation.

These ideas are consistent with a study of patient and provider attitudes towards the use of financial incentives to support diabetes self-management [15].

\subsection{Setting and Study Population}

DaVita HealthCare Partners is a very large integrated care organization delivering care in the southern California region to over half a million commercial, Medicare and Medicaid enrolled members. Over 60 medical clinics in the greater Los Angeles and Orange County area and nearly as many hospitals serve these members (see https://healthcarepartners.com/Locations/).

Patients were identified as having a diabetes diagnosis using the DHCP diabetes registry. Patients were excluded if 1 ) age $<18$ years, 2) type 1 diabetes diagnosis, 3) gestational diabetes diagnosis, 4) dementia diagnosis or 5) if their last A1c value indicated glycemic control. For the last criteria, patients age 65 and older were eligible for the DGA if their most recent A1c was equal to or greater than $9.0 \%$ and $8.0 \%$ for those under age 65 . Given diabetes treatment standards these values would indicate each patient had an A1c value at least $1.0 \%$ over their likely A1c goal [3].

\section{Study Design and Analysis}

In order to evaluate the impact of financial incentives and the DGA, patients 
who met the above criteria were randomly assigned to one of three groups: 1) no DGA, 2) DGA with no financial incentives (non-incentive) or 3) DGA with financial incentives (incentive DGA). An initial goal of 950 patients or 316 per arm was estimated to provide the research team a large enough sample to detect differences in A1c levels of $0.5 \%$ and was considered feasible by DHCP. The lead health educator at a participating site was provided a list of patients identified for the non-incentive and incentive DGA. The written DGA invitation letters and follow-up phone calls were edited to include description of the financial incentives. Nine locations within four regions were identified for participation due to the diversity of patients as well as familiarity with DGAs and necessary staffing levels. The first DGA launched in June 2012 and the last during June 2013 to ensure at least six months of follow-up. The full study period lasted for 18 months, from June 2012 through December 2013.

The USC research team acted as consultants only regarding research design and post intervention analysis. No one outside of DHCP had access to any personlevel confidential health data or interacted with participants during the project. DHCP providers and staff delivered patient-centered care according to current standards of care. Given the limited involvement of the research team, analyses are descriptive as presented under the Results section and the implications of these results and implementation challenges are reviewed in detail in the Discussion, Lessons and Conclusions section.

\section{Results}

As shown in Table 1, 1146 patients were reached via phone and invited to participate in either a non-incentive DGA or DGA with financial incentives. At the

Table 1. Diabetes Group Appointment (DGA) Enrollment by Site and Type: Non-Incentive and Financial Incentive.

\begin{tabular}{ccccc}
\hline \multirow{2}{*}{ Site } & \multicolumn{2}{c}{ Number of Patients Contacted } & \multicolumn{2}{c}{ Total Enrollment } \\
\cline { 2 - 5 } & Non-Incentive DGA & Incentive DGA & Non-Incentive DGA (\%) & Incentive DGA (\%) \\
\hline 1.1 & 82 & 45 & $24(29.3)$ & $13(28.9)$ \\
$1.2^{\mathrm{a}}$ & 73 & 80 & $18(24.7)$ & $15(18.8)$ \\
$1.3^{\mathrm{b}}$ & 35 & 25 & $13(37.1)$ & $16(64.0)$ \\
2.1 & 82 & 52 & $18(22.0)$ & $19(36.5)$ \\
2.2 & 104 & 62 & $21(20.2)$ & $14(22.6)$ \\
2.3 & 62 & 35 & $18(29.0)$ & $14(40.0)$ \\
2.4 & 86 & 33 & $8(9.3)$ & $12(36.4)$ \\
3.1 & 123 & 74 & $26(21.1)$ & $19(25.7)$ \\
4.1 & 68 & 25 & $15(22.1)$ & $16(64.0)$ \\
Total & 685 & 461 & $161(23.5)$ & $138(29.9)$ \\
\hline
\end{tabular}

a. At this site, when staff had difficulty with recruitment, they bypassed sending an invitation letter prior to calling patients. b. Spanish-speaking DGA. 
height of enrollment, 161 patients were participating in a non-incentive DGA and 138 patients in an incentive DGA. Recruitment by site and type of DGA varied substantially, from a low of $9.3 \%$ to a high of $64.0 \%$. Overall, $23.5 \%$ of patients invited to a non-incentive DGA, who were reached via phone, attended at least one DGA, versus $29.9 \%$ of patients reached for an incentive DGA. The most common reason for declining the DGA, either incentive or non-incentive, was work ( $45 \%$ of respondents). We use the term reached, because many more patients (additional 497) were identified for enrollment in either a non-incentive or incentive DGA, but due to an invalid phone number or inability to connect with these patients after at least three attempts, staff would proceed to the next patient on their list. In order to minimize the potential impact of timing differences each site had the goal of launching a non-incentive and incentive DGA within one month of each other, but this proved challenging. Six sites had the two types of DGAs launch within three months of each other, but in the case of the other three sites, the start dates ranged from 6 - 10 months.

Table 2 provides descriptive statistics for patients enrolled in the non-incentive and incentive DGAs who responded to a self-administered questionnaire offered at the end of the first DGA meeting. The mean age of attendees was comparable at about 61 years. Women comprised a greater proportion of the non-incentive DGAs than the incentive DGAs (61.8\% vs. $51.0 \%$, respectively). Slightly more African American patients (17.0\% vs. $11.3 \%$ ), those with a high school education or less $(49.0 \%$ vs. $42.3 \%)$, and persons who preferred not to check a box indicating their annual family income (17.3\% vs. $9.5 \%)$ were enrolled in non-incentive relative to incentive DGAs, respectively. Comparable values are not available for those patients who were randomized to the no DGA comparison group as this information was not routinely collected during 2012-2013.

The main outcome of interest, whether financial incentives actually resulted in better glycemic control in type 2 diabetes patients is shown in Table 3. The patients enrolled in a DGA with financial incentives lowered their Alc level more on average more than those patients enrolled in a non-incentive DGA or no DGA $(-1.7 \%$ vs. $-0.7 \%$ vs. $-0.4 \%$, respectively). While the mean starting A1c was relatively close among those patients attending the financial incentive and non-incentive DGAs, those patients who were not invited to a DGA as part of the study tended to have a lower starting A1c level on average (9.9\% vs. $9.7 \%$ vs. $9.1 \%$, respectively).

\section{Discussion, Lessons and Conclusions}

The ADA has recognized and recommended the role of diabetes self-management. To that end, DHCP primary care teams developed and implemented the DGA intervention as part of their commitment to delivering high quality evidencebased diabetes care. Our results show that financial incentives within a large integrated managed care organization are feasible, and suggest a positive influence on both DGA attendance and Alc levels. Additional research with larger samples 
Table 2. Patient Self-Reported Sociodemographic Characteristics by Diabetes Group Appointment (DGA) Type: Non-Incentive and Financial Incentive.

\begin{tabular}{|c|c|c|c|}
\hline Characteristic & $\begin{array}{l}\text { Total DGA Patients } \\
(\mathrm{N}=187 \text { to } 212)\end{array}$ & $\begin{array}{l}\text { Non-Incentive DGA } \\
\qquad(\mathrm{N}=91 \text { to } 110)\end{array}$ & $\begin{array}{l}\text { Incentive DGA } \\
(\mathrm{N}=95 \text { to } 102)\end{array}$ \\
\hline Mean Age (range) & 61.4 years ( 31 to 90 ) & 61.7 years ( 31 to 88 ) & 61.1 years ( 31 to 90$)$ \\
\hline \multicolumn{4}{|l|}{ Gender } \\
\hline Female & $56.6 \%$ & $61.8 \%$ & $51.0 \%$ \\
\hline Male & $43.4 \%$ & $38.2 \%$ & $49.0 \%$ \\
\hline \multicolumn{4}{|l|}{ Race/Ethnicity } \\
\hline Non-Hispanic White & $20.8 \%$ & $19.0 \%$ & $22.7 \%$ \\
\hline African American & $14.2 \%$ & $17.0 \%$ & $11.3 \%$ \\
\hline Hispanic & $54.8 \%$ & $52.0 \%$ & $57.7 \%$ \\
\hline Asian & $5.6 \%$ & $7.0 \%$ & $4.1 \%$ \\
\hline Other & $4.6 \%$ & $5.0 \%$ & $4.1 \%$ \\
\hline \multicolumn{4}{|l|}{ Education Level } \\
\hline High School or less & $45.7 \%$ & $49.0 \%$ & $42.3 \%$ \\
\hline Some College & $35.7 \%$ & $32.4 \%$ & $39.2 \%$ \\
\hline College and above & $18.6 \%$ & $18.6 \%$ & $18.6 \%$ \\
\hline \multicolumn{4}{|l|}{ Annual Family Income } \\
\hline$<\$ 15,000$ & $16.6 \%$ & $16.3 \%$ & $16.8 \%$ \\
\hline$\$ 15,000$ to $\$ 24,999$ & $11.9 \%$ & $15.3 \%$ & $8.4 \%$ \\
\hline$\$ 25,000$ to $\$ 34,999$ & $11.9 \%$ & $12.2 \%$ & $11.6 \%$ \\
\hline$\$ 35,000$ to $\$ 49,999$ & $10.9 \%$ & $10.2 \%$ & $11.6 \%$ \\
\hline$\$ 50,000$ to $\$ 74,999$ & $14.5 \%$ & $11.2 \%$ & $17.9 \%$ \\
\hline$\$ 75,000$ to $\$ 99,999$ & $11.4 \%$ & $9.2 \%$ & $13.7 \%$ \\
\hline$\geq \$ 100,000$ & $6.2 \%$ & $6.1 \%$ & $6.3 \%$ \\
\hline Don’t Know & $3.1 \%$ & $2.0 \%$ & $4.2 \%$ \\
\hline Prefer Not to Say & $13.5 \%$ & $17.3 \%$ & $9.5 \%$ \\
\hline
\end{tabular}

a. Sample size varies due to the response rate to each question, which were part of a self-administered written questionnaire given as part of a satisfaction survey at the end of the first DGA meeting.

Table 3. Hemoglobin A1c by Diabetes Group Appointment (DGA) Type: No DGA, Non-Incentive and Financial Incentive.

\begin{tabular}{cccc}
\hline & \multicolumn{3}{c}{ DGA Type } \\
\cline { 2 - 4 } & No DGA & Non-Incentive DGA & Incentive DGA \\
\hline Sample Size & 254 & 161 & 138 \\
Mean Starting A1c & $9.1 \%$ & $9.7 \%$ & $9.9 \%$ \\
Mean Ending A1c & $8.7 \%$ & $9.0 \%$ & $8.7 \%$ \\
Difference & $-0.4 \%$ & $-0.7 \%$ & $-1.2 \%$ \\
\hline
\end{tabular}


is desirable to confirm our observations.

An unintended set of findings emerged from the study and provide valuable lessons for the applied evaluation literature and primary care community. These findings relate to the complexities of implementing "live" interventions based on rigorous, scientific designs. Researchers were challenged to evaluate the interventions being delivered in "real time" without the ability to manage or know all aspects of implementation.

Operationalization of a standardized DGA is one example. The project manager conducted site visits and, along with the lead health educator met monthly with staff implementing the DGAs to increase uniformity, but with different lead physicians, nurses, dieticians, and educators, each site sometimes made last minute adjustments due to their personal practice styles and comfort levels or had to due to resource constraints (i.e. availability of a large enough room, down two primary care providers). For example, the lead doctor at two study sites met with patients one-on-one during the DGA, which was not standard practice at other sites, but this was his preference. Also, per the request of one sites' dietitian and nurse, patients on intensive insulin therapy (basal, plus bolus insulin therapy) were excluded from participation.

With respect to consistent launch dates, as previously mentioned, the start of different DGAs, both within and across sites, occurred at different times over a 12-month period and as a result length of follow-up also varied. Time of year may impact attendance. Patients may miss appointments more in summer months due to vacation and family events (e.g., weddings) or because of holidays in November and December. Therefore, differences in attendance within sites may relate more to differences in the DGA start dates than due to financial incentives.

Another internal challenge relates to the diverse socio-demographic characteristics of DHCP members, reflective of Los Angeles. The health literacy of some patients was relatively low (e.g., difficulty reading and completing self-administered questionnaires), creating another barrier to diabetes education and self-management. In this study, $46 \%$ of DGA patients had a high school education or less. Low health literacy has been associated with misperceptions of A1c control [16].

The appointment times of the DGA were also difficult to plan. The most common reason patients gave who declined to participate was that the proposed times were inconvenient. The DGAs were offered only in the mornings on a weekday due to primary care team availability and because this reflected prior DHCP experience with DGAs. Patients who declined to attend a DGA, but indicated an interest, suggested an evening or weekend time. Current DGA participants also suggested having evening and weekend times available. Now that experience has been gained with the DGAs across several sites and regions, some primary care teams may feel comfortable transitioning to or adding an evening or weekend DGA.

Lastly, the project manager invited all DGA lead physicians to provide feed- 
back. Five physicians were interviewed in person. While all five physicians agreed the DGA was valuable, because they felt the education and support was necessary, the challenge of "getting patients through the door" as well as convincing them of the need for change was reiterated. With respect to the latter issue, it was noted that DGA attendance might trigger the prescription of insulin, but that patients could still be resistant to taking medication. While concern arose that patients might be coming to the incentive DGAs for the "wrong" reason (i.e., for the extrinsic motivator of money versus the intrinsic desire to improve health) the overall view was that the incentives helped to engage patients, but weren't enough to overcome the barrier of a fulltime job and that "nothing lasts," so motivators need to be changing, especially when the behavior changes are meant to last a lifetime. Consistent with the initial concerns of providers and staff to avoid the use of penalties or threat of a loss, a few physicians and staff noted that a handful of patients purposefully missed DGAs because of embarrassment. Either that they feared their A1c level did not improve or in the case of DGA meetings focused on exercise (including yoga) that they wouldn't be able to complete the exercise.

\section{Limitations}

Implementation challenges limit our ability to assess the impact of the DGAs and financial incentives. In order to protect patient confidentiality, no researcher had access to the raw data and ultimately what we report is descriptive. While a randomized controlled trial may be the gold standard of research designs, logistical challenges within DHCP and beyond DHCP's control preclude knowing whether confounding variables (e.g., patients on insulin and adherence, access to other services such as worksite wellness benefits) influenced outcomes.

In summary, this study seeks to build upon the emerging behavioral economics literature as it applies to health care and impacting health behavior. The results are two-fold and show that use of financial incentives is feasible within a large integrated managed care organization, and suggest a positive influence on both DGA attendance and reducing Alc levels. The second, unexpected set of findings includes a summary of practical and logistical challenges to guide future efforts in implementing and evaluating behavioral change interventions.

\section{Acknowledgements}

The authors gratefully acknowledge the work of Rachel Gordon, project manager and Aurora Galindo Simental, health educator/promotora.

\section{References}

[1] Centers for Disease Control and Prevention (2017) National Diabetes Statistics Report, 2017. 1-20.

[2] Babey, S.H., et al. (2016) Prediabetes in California: Nearly Half of California Adults on Path to Diabetes. Policy Brief UCLA Cent Health Policy Res, PB2016-1, 1-8. 
[3] American Diabetes Association (2017) Standards of Medical Care in Diabetes-2017. Diabetes Care, 40, S1-S142.

[4] Beck, J., et al. (2017) National Standards for Diabetes Self-Management Education and Support. Diabetes Care, 40, 1409-1419. https://doi.org/10.2337/dci17-0025

[5] Burke, R.E. and O’Grady, E.T. (2012) Group Visits Hold Great Potential for Improving Diabetes Care and Outcomes, But Best Practices Must Be Developed. Health Affairs (Millwood), 31, 103-109. https://doi.org/10.1377/hlthaff.2011.0913

[6] Kirsh, S., Watts, S., Pascuzzi, K., O’Day, M.E., Davidson, D., Strauss, G., Kern, E.O. and Aron, D.C. (2007) Shared Medical Appointments Based on the Chronic Care Model: A Quality Improvement Project to Address the Challenges of Patients with Diabetes with High Cardiovascular Risk. BMJ Quality \& Safety, 16, 349-353.

https://doi.org/10.1136/qshc.2006.019158

[7] Stults, C.D., et al. (2016) Shared Medical Appointments: A Promising Innovation to Improve Patient Engagement and Ease the Primary Care Provider Shortage. Population Health Management, 19, 11-16. https://doi.org/10.1089/pop.2015.0008

[8] Lorincz, I.S., Lawson, B.C. and Long, J.A. (2013) Provider and Patient Directed Financial Incentives to Improve Care and Outcomes for Patients with Diabetes. Current Diabetes Reports, 13, 188-195. https://doi.org/10.1007/s11892-012-0353-9

[9] Kullgren, J.T., Hafez, D., Fedewa, A. and Heisler, M. (2017) A Scoping Review of Behavioral Economic Interventions for Prevention and Treatment of Type 2 Diabetes Mellitus. Current Diabetes Reports, 17, 73.

https://doi.org/10.1007/s11892-017-0894-Z

[10] Long, J.A., et al. (2012) Peer Mentoring and Financial Incentives to Improve Glucose Control in African American Veterans: A Randomized Trial. Annals of Internal Medicine, 156, 416-424. https://doi.org/10.7326/0003-4819-156-6-201203200-00004

[11] Misra-Hebert, A.D., Hu, B., Taksler, G., Zimmerman, R. and Rothberg, M.B. (2016) Financial Incentives and Diabetes Disease Control in Employees: A Retrospective Cohort Analysis. Journal of General Internal Medicine, 31, 871-877. https://doi.org/10.1007/s11606-016-3686-2

[12] McCuistion, M.H., Stults Cheryl, D., Dohan, D., Frosch Dominick, L., Hung Dorothy, Y. and Tai-Seale, M. (2014) Overcoming Challenges to Adoption of Shared Medical Appointments. Population Health Management, 17, 100-105. https://doi.org/10.1089/pop.2013.0035

[13] Farina, K. (2013) Can Financial Incentives Improve Self-Management Behaviors? American Journal of Managed Care, 19, E8.

[14] Kroenke, K., Spitzer, R.L. and Williams, J.B. (2001) The PHQ-9: Validity of a Brief Depression Severity Measure. Journal of General Internal Medicine, 16, 606-613. https://doi.org/10.1046/j.1525-1497.2001.016009606.x

[15] Blondon, K., Klasnja, P., Coleman, K. and Pratt, W. (2014) An Exploration of Attitudes toward the Use of Patient Incentives to Support Diabetes Self-Management. Psychology \& Health, 29, 552-563. https://doi.org/10.1080/08870446.2013.867346

[16] Ferguson, M.O., et al. (2015) Low Health Literacy Predicts Misperceptions of Diabetes Control in Patients with Persistently Elevated A1C. The Diabetes Educator, 41, 309-319. https://doi.org/10.1177/0145721715572446 